\title{
Silencing of long non-coding RNA NCK1-AS1 inhibits cell proliferation and migration via inhibition of microRNA-134 in cervical cancer
}

\author{
LU HUANG, XIAOQIN GAN, LI HE, LUYING WANG and JIE YU \\ Department of Obstetrics and Gynaecology, Chengdu Women and Children's Central Hospital, \\ Chengdu, Sichuan 610031, P.R. China
}

Received September 16, 2018; Accepted April 26, 2019

DOI: $10.3892 /$ etm.2019.7799

\begin{abstract}
Long non-coding RNA NCK1-antisense 1 (AS1) has recently been demonstrated to promote cell proliferation and induce cell cycle progression through the crosstalk NCK1-AS1/microRNA (miR)-6857/cyclin dependent kinase 1 pathway in cervical cancer. However, the regulatory mechanism of NCK1-AS1 in cervical cancer growth and metastasis remains largely unclear. In the present study, it was identified that NCK1-AS1 was significantly upregulated in cervical cancer tissues compared with the levels in adjacent non-tumour tissues. High expression levels of NCK1-AS1 were associated with tumour progression and poor prognosis in patients with cervical cancer. Silencing of NCK1-AS1 expression significantly decreased the levels of proliferation and migration of cervical cancer cells, and decreased the protein expression levels of matrix metalloproteinase (MMP)-2 and MMP-9. The results of the luciferase reporter gene assay indicated that there was an miR-134 binding site within the NCK1-AS1 gene in cervical cancer cells. miR-134 was significantly downregulated in cervical cancer tissues compared with the miR-134 levels in adjacent non-tumour tissues, and the expression level of miR-134 was inversely correlated with the NCK1-AS1 expression levels in cervical cancer tissues. Knockdown of miR-134 attenuated the inhibitory effects of NCK1-AS1 downregulation on the proliferation and migration of cervical cancer cells. Therefore, the data from the present study suggested that NCK1-AS1 serves a promotive role in cervical cancer cell proliferation and migration by functioning as a molecular sponge for miR-134. NCK1-AS1 may become a novel therapeutic target for cervical cancer.
\end{abstract}

Correspondence to: $\mathrm{Dr}$ Jie Yu, Department of Obstetrics and Gynaecology, Chengdu Women and Children's Central Hospital, 1617 Riyue Avenue, Chengdu, Sichuan 610031, P.R. China

E-mail: haloyujie@sina.com

Key words: NCK1-antisense RNA 1, cervical cancer, microRNA-134, proliferation, migration

\section{Introduction}

Cervical cancer is one of the most common malignancies in females worldwide, with 500,000 incident cases and 300,000 mortalities each year; it is commonly caused by infection with high-risk strains of human papillomavirus (1-3). Despite improvements in surgical resection combined with radiotherapy and chemotherapy, the prognosis of patients with advanced cervical cancer remains poor, primarily due to metastasis and drug tolerance $(2,4,5)$. Therefore, it is critically important to explore the molecular mechanism underlying cervical cancer progression, which may assist in developing novel and efficient biomarkers and therapeutic targets.

Long non-coding RNAs (lncRNAs) are a group of single-strand non-coding RNAs measuring $>200$ nucleotides in length, which comprise $\sim 80 \%$ of non-coding RNAs $(6,7)$. In previous years, accumulating evidence has revealed that lncRNAs serve key roles in a variety of cellular biological processes, including cell proliferation, apoptosis, differentiation, viability, autophagy, migration and angiogenesis (8-10). In addition, a large number of lncRNAs have been demonstrated to serve promotive or suppressive roles in various types of human cancer, including cervical cancer, through interactions with the downstream microRNAs (miRNAs) or proteins, and certain lncRNAs are associated with cancer recurrence and poor prognosis $(11,12)$. For example, lncRNA X inactive specific transcript accelerates cervical cancer progression by upregulating FUS RNA binding protein by competitively binding with miR-200a (13). The lncRNA Taurine up-regulated 1 promotes cervical cancer progression by regulating the miR-138-5p-sirtuin 1 axis (14). Recently, Li et al (15) suggested that 1ncRNA NCK1-antisense 1 (AS1) promoted cervical cancer cell proliferation by inducing cell cycle progression by regulating the expression of miR-6857 and cyclin dependent kinase 1 . However, the underlying molecular mechanism of NCK1-AS1 in cervical cancer growth and metastasis remains largely unclear.

miRNAs, a group of small non-coding RNAs measuring 22-25 nucleotides in length, are gene expression regulators that serve key roles in cancer development and progression $(15,16)$. Among the cancer-associated miRNAs, miR-134 has been demonstrated to be essential for cancer development and 
progression, as it participates in cancer cell proliferation, apoptosis, migration, invasion and drug resistance $(17,18)$. However, to the best of our knowledge, the function of miR-134 in cervical cancer, and the association between miR-134 and NCK1-AS1, has not been described previously.

The present study aimed to examine the expression of NCK1-AS1 in cervical cancer tissues and cell lines, and to investigate the association between NCK1-AS1 expression and clinicopathological characteristics in cervical cancer. In addition, in vitro experiments were performed to explore the regulatory mechanism of NCK1-AS1 in the proliferation and migration of cervical cancer cells involving miR-134.

\section{Materials and methods}

Tissue collection. The present study was approved by the Ethics Committee of Chengdu Women and Children's Central Hospital (Chengdu, China). A total of 52 pairs of cervical cancer tissues and adjacent normal tissues were collected from patients with primary cervical cancer at Chengdu Women and Children's Central Hospital between March 2010 and September 2012. Written informed consent was obtained from all participants. None of the patients had received chemotherapy or radiotherapy prior to surgery. The cervical cancer tissues and adjacent normal tissues were rapidly frozen in liquid nitrogen shortly following surgical resection and stored until use. The clinical characteristics of the patients are summarized in Table I.

Cell culture and transfection. A normal human cervix epithelial Ect1/E6E7 cell line and cervical cancer SiHa, HeLa, C-33A and CaSki cell lines were purchased from the American Type Culture Collection. The cells were cultured in Dulbecco's modified Eagle's medium (DMEM; Thermo Fisher Scientific, Inc.) containing $10 \%$ foetal bovine serum (Thermo Fisher Scientific, Inc.) at $37^{\circ} \mathrm{C}$ in an incubator with $5 \% \mathrm{CO}_{2}$. Cell transfection was performed with $100 \mathrm{nM}$ of negative control (NC) small interfering RNA (siRNA; cat. no. 4390843; Thermo Fisher Scientific, Inc.), NCK1-AS1 siRNA (cat. no. 4392420; Thermo Fisher Scientific, Inc.), NC inhibitor (cat. no. 4464076; Thermo Fisher Scientific, Inc.) or miR-134 inhibitor (cat. no. 4464084; Thermo Fisher Scientific, Inc.) using Lipofectamine ${ }^{\circledR} 2000$ (Invitrogen; Thermo Fisher Scientific, Inc.) according to the manufacturer's protocol. The following experiments were conducted $48 \mathrm{~h}$ after transfection.

Reverse transcription quantitative polymerase chain reaction $(R T-q P C R)$. Total RNA was extracted from tissues and cells using TRIzol ${ }^{\circledR}$ reagent (Thermo Fisher Scientific, Inc.) according to the manufacturer's protocol. RNA $(1 \mu \mathrm{g})$ was then reverse transcribed into cDNA using the miScript Reverse Transcription kit (Qiagen $\mathrm{GmbH}$ ), according to the manufacturer's protocol. Then, qPCR was performed using cDNA with a SYBR ${ }^{\circledR}$ Green Real-Time PCR Master Mix (Thermo Fisher Scientific, Inc.), following the manufacturer's protocol. The qPCR thermocycler conditions were as follows: $95^{\circ} \mathrm{C}$ for $3 \mathrm{~min}$, followed by 45 cycles of $95^{\circ} \mathrm{C}$ for $15 \mathrm{sec}, 60^{\circ} \mathrm{C}$ for $15 \mathrm{sec}$ and $72^{\circ} \mathrm{C}$ for $15 \mathrm{sec}$. GAPDH and U6 were used as internal references. The $2^{-\Delta \Delta \mathrm{Cq}}$ method was used to analyse the relative gene expression (19). The following primer sequences were used: GAPDH forward, 5'-CTGGGCTACACTGAG CACC-3' and reverse, 5'-AAGTGGTCGTTGAGGGCA ATG-3'; U6 forward, 5'-CTCGCTTCGGCAGCACA-3' and reverse, 5'-AACGCTTCACGAATTTGCGT-3'. The primers for NCK1-AS1 (cat. no. Hs00606619_CE) and miR-134 (cat. no. Hs00500260_CE) were purchased from Thermo Fisher Scientific, Inc.

Cell Counting Kit-8 (CCK-8) assay. The transfected HeLa and SiHa cells $\left(3 \times 10^{3}\right.$ cells/well) were seeded into 96 -well plates and cultured at $37^{\circ} \mathrm{C}$ with $5 \% \mathrm{CO}_{2}$ for $0,24,48$ and $72 \mathrm{~h}$. Then, CCK-8 solution (Dojindo Molecular Technologies) was added, and cells were incubated at $37^{\circ} \mathrm{C}$ for $2 \mathrm{~h}$. Subsequently, the absorbance value was determined at $450 \mathrm{~nm}$ using a microplate reader (Bio-Rad Laboratories, Inc.).

Wound healing assay. The transfected HeLa and SiHa cells were seeded into 24 -well plates and cultured at $37^{\circ} \mathrm{C}$ with $5 \% \mathrm{CO}_{2}$ to $100 \%$ confluence. Then, the cells were scratched with a sterile $200 \mu \mathrm{l}$ pipette tip and washed twice with PBS. Cells in DMEM were then incubated at $37^{\circ} \mathrm{C}$ with $5 \% \mathrm{CO}_{2}$ for $24 \mathrm{~h}$. Images were captured at 0 and $24 \mathrm{~h}$ using a light microscope (magnification, x40).

Luciferase reporter gene assay. The target miRNAs of NCK1-AS1 were predicted using RNAhybrid 2.12 software (http://bibiserv.techfak.uni-bielefeld.de/rnahybrid/). miR-134 was predicted to have binding sites in the NCK1-AS1 gene. To confirm this prediction, wild-type (WT) and mutant (Mut) NCK1-AS1 sequences were produced by Shanghai GenePharma Co., Ltd., and inserted into the pGL3 luciferase vector (Promega Corporation). HeLa and SiHa cells were transfected with miR-134 mimics or miR-NC, together with WT or Mut pGL3-NCK1-AS1 reporter plasmids, using Lipofectamine ${ }^{\circledR} 2000$ according to the manufacturer's protocol. At $48 \mathrm{~h}$ after transfection, luciferase activities were determined using a Dual Luciferase Reporter Assay kit (Promega Corporation) in accordance with the manufacturer's protocol. Renilla luciferase activity was normalized to that of firefly luciferase.

Western blot analysis. Transfected cells were lysed in cold radioimmunoprecipitation assay lysis buffer (Thermo Fisher Scientific, Inc.). The protein concentration was determined using the BCA Protein Assay kit (Thermo Fisher Scientific, Inc.) in accordance with the manufacturer's protocol. Proteins (50 $\mu \mathrm{g} /$ lane) were separated with $10 \%$ SDS-PAGE and transferred to polyvinylidene fluoride membranes (Thermo Fisher Scientific, Inc.). The membranes were incubated in 5\% non-fat dried milk (Yili Group) in PBS at $4^{\circ} \mathrm{C}$ overnight. Following incubation with rabbit anti-human matrix metalloproteinase (MMP)-2 antibody (1:500; cat. no. ab37150; Abcam), rabbit anti-human MMP-9 antibody (1:200; cat. no. ab73734; Abcam) or rabbit anti-human GAPDH antibody (1:500; cat. no. ab9485; Abcam) at room temperature for $3 \mathrm{~h}$, the membranes were incubated with horseradish peroxidase-conjugated goat anti-rabbit secondary antibody (1:5,000; cat. no. ab6721; Abcam) at room temperature for $1 \mathrm{~h}$. An ECL Western Blotting kit (Thermo Fisher Scientific, Inc.) was used to detect the immune complexes. Image-Pro Plus 
Table I. Association between lncRNA NCK1-AS1 expression and clinicopathological characteristics of patients with cervical cancer.

\begin{tabular}{|c|c|c|c|c|}
\hline \multirow[b]{2}{*}{ Variables } & \multirow[b]{2}{*}{ Cases $(n=52)$} & \multicolumn{2}{|c|}{ NCK1-AS1 level } & \multirow[b]{2}{*}{ P-value } \\
\hline & & Low $(n=26)$ & High $(n=26)$ & \\
\hline Age, years & & & & 0.776 \\
\hline$<55$ & 20 & 9 & 11 & \\
\hline$\geq 55$ & 32 & 17 & 15 & \\
\hline Tumor size, $\mathrm{cm}$ & & & & 0.083 \\
\hline$\leq 4$ & 33 & 20 & 13 & \\
\hline$>4$ & 19 & 6 & 13 & \\
\hline Differentiation & & & & 0.173 \\
\hline Well-Moderate & 41 & 23 & 18 & \\
\hline Poor & 11 & 3 & 8 & \\
\hline Clinical stage & & & & 0.003 \\
\hline I-II & 33 & 22 & 11 & \\
\hline III-IV & 19 & 4 & 15 & \\
\hline Lymph node metastasis & & & & 0.006 \\
\hline No & 36 & 23 & 13 & \\
\hline Yes & 16 & 3 & 13 & \\
\hline Distant metastasis & & & & 0.051 \\
\hline No & 47 & 26 & 21 & \\
\hline Yes & 5 & 0 & 5 & \\
\hline
\end{tabular}

NCK1-AS1, NCK1-antisense 1.

software 6.0 (Media Cybernetics, Inc.) was used to analyse the relative protein expression.

Statistical analysis. Statistical analysis was performed using SPSS 19.0 software (IBM Corp.). The data are expressed as the mean \pm standard deviation. An unpaired Student's t-test was used to analyse differences between two groups. A one-way analysis of variance and Turkey's post-hoc test was used for multiple comparisons. The $\chi^{2}$ test was performed to analyse the association between NCK1-AS1 expression and the clinical features of patients. A Kaplan-Meier survival curve and a log-rank test were applied for survival analysis. Spearman's correlation analysis was conducted to evaluate the correlation between NCK1-AS1 and miR-134 expression in cervical cancer tissues. $\mathrm{P}<0.05$ was considered to indicate a statistically significant difference.

\section{Results}

Upregulation of NCK1-AS1 in cervical cancer. In the present study, NCK1-AS1 expression was first analysed in several human cervical cancer cell lines and normal cells using the qPCR analysis. As demonstrated in Fig. 1A, NCK1-AS1 was significantly upregulated in cervical cancer cells compared with the NCK1-AS1 levels in Ect1/E6E7 cells. To additionally confirm the upregulation of NCK1-AS1 in cervical cancer, a total of 52 paired cervical cancer tissues and adjacent normal tissues were analysed. The qPCR analysis was then performed to examine NCK1-AS1 expression. As indicated in Fig. 1B, the expression levels of NCK1-AS1 were increased in cervical cancer tissues compared with the expression levels in adjacent normal tissues. Then, the clinical significance of NCK1-AS1 expression in cervical cancer was studied. Patients were then divided into high and low NCK1-AS1 expression groups, using its mean expression value as the cut-off (2.44). As indicated in Table I, a high expression NCK1-AS1 level was significantly associated with positive lymph node metastasis and advanced clinical stage. In addition, it was identified that the patients with cervical cancer with high NCK1-AS1 expression exhibited a shorter survival times compared with patients with low NCK1-AS1 expression levels (Fig. 1C). These data suggest that NCK1-AS1 may be involved in the malignant progression of cervical cancer.

Silencing of NCK1-AS1 expression inhibits the proliferation and migration of cervical cancer cells. The function of NCK1-AS1 in cervical cancer was studied using HeLa and SiHa cells. These 2 cell lines were transfected with NCK1-AS1 siRNA to knock down the expression of NCK1-AS1. Transfection with NC siRNA was performed as a control. qPCR data indicated that the expression of NCK1-AS1 was significantly downregulated following transfection with NCK1-AS1 siRNA compared with the expression in the NC siRNA group (Fig. 2A and B). The CCK-8 and wound healing assays additionally indicated that the knockdown of NCK1-AS1 significantly decreased the proliferation and migration of HeLa and SiHa cells (Fig. 2C-F), indicating that 

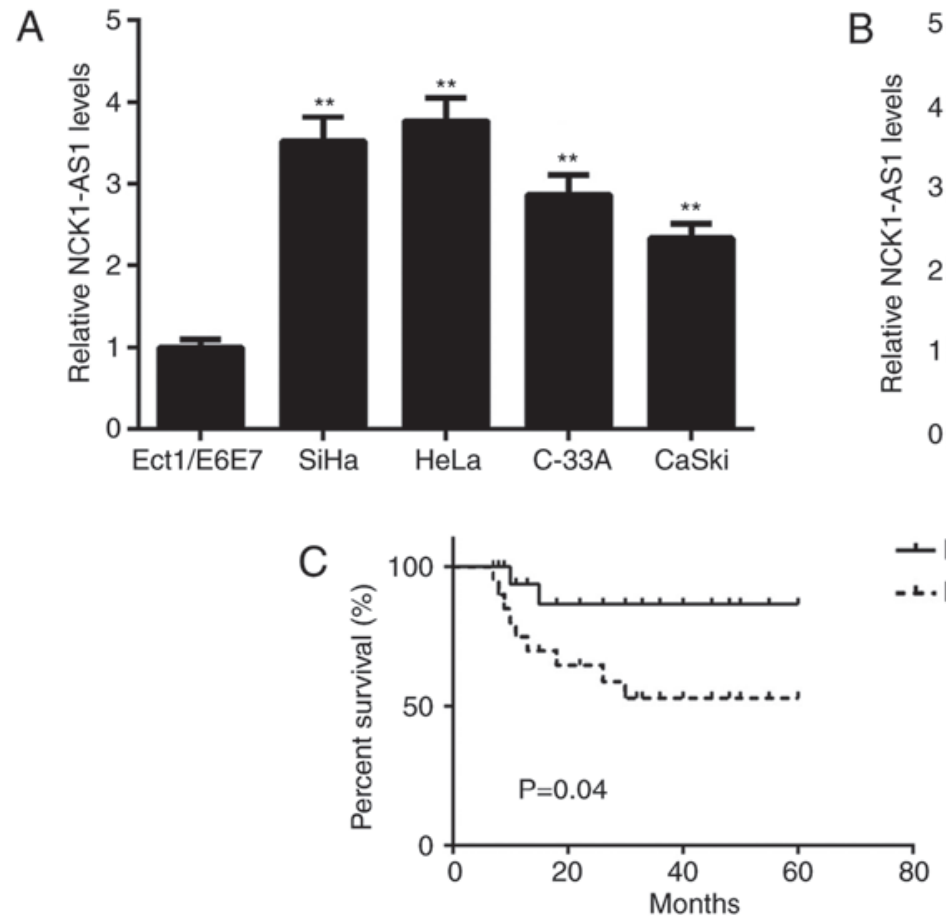

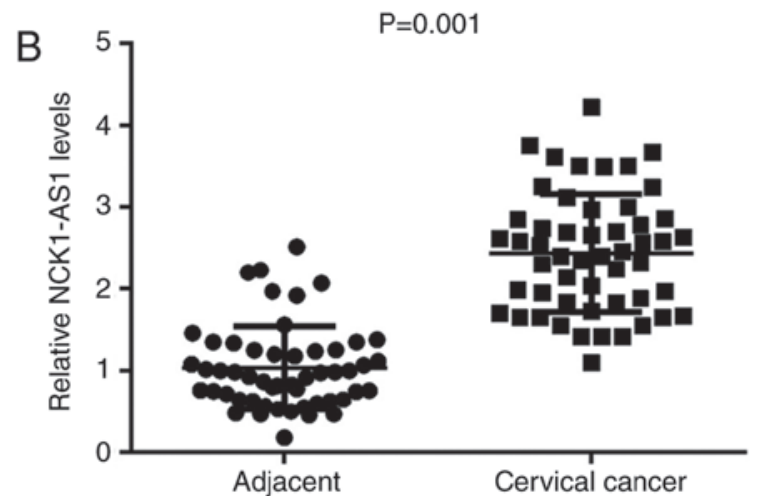

- Low NCK1-AS1 expression

- ᄂ. High NCK1-AS1 expression

Figure 1. Upregulation of NCK1-AS1 in cervical cancer. (A) The NCK1-AS1 levels were significantly upregulated in cervical cancer cell lines compared with the NCK1-AS1 levels in Ect1/E6E7 cells. ${ }^{* *} \mathrm{P}<0.01$ vs. Ect1/E6E7. (B) NCK1-AS1 was also significantly upregulated in cervical cancer tissues compared with NCK1-AS1 levels in adjacent normal tissues. (C) The patients with cervical cancer with high NCK1-AS1 expression exhibited a shorter survival time compared with patients with a low expression of NCK1-AS1. NCK1-AS1, NCK1-antisense 1.

NCK1-AS1 may serve a promotive role in cervical cancer cell proliferation and migration. In addition, it was observed that silencing of NCK1-AS1 significantly decreased the protein expression of MMP-2 and MMP-9, 2 important markers for tumor cell migration and invasion (Fig. $2 \mathrm{G}$ and $\mathrm{H}$ ).

NCK1-AS1 gene contains an miR-134 binding site in cervical cancer cells. Bioinformatic analysis was then performed to study the potential NCK1-AS1-miR interactions. As indicated in Fig. 3A, miR-134 had a potential binding site in NCK1-AS1. To clarify this prediction, a luciferase reporter gene assay was performed using cervical cancer cells. The data demonstrated that transfection with miR-134 mimics led to a significant decrease in the luciferase activity of the WT NCK1-AS1 luciferase reporter gene plasmid but did not affect the luciferase activity of the Mut NCK1-AS1 luciferase reporter gene plasmid (Fig. 3B and C). In addition, it was identified that silencing of NCK1-AS1 expression caused a significant upregulation of miR-134 in cervical cancer cells (Fig. 3D and E). These results suggested that NCK1-AS1 may directly target miR-134 in cervical cancer cells.

Downregulation of miR-134 is inversely correlated with the upregulation of NCK1-AS1 in cervical cancer tissues. It was identified that the expression levels of miR-134 were significantly decreased in cervical cancer cells compared with the expression levels in Ect1/E6E7 cells (Fig. 4A). Consistently, miR-134 expression levels were also significantly downregulated in cervical cancer tissues compared with the expression levels in adjacent tissues (Fig. 4B). Notably, an inverse correlation between miR-134 and NCK1-AS1 expression was observed in cervical cancer tissues (Fig. 4C). Therefore, the upregulation of NCK1-AS1 may contribute to the downregulation of miR-134 in cervical cancer.

Silencing of miR-134 expression impairs the suppressive effects of NCK1-AS1 inhibition on cervical cancer cell proliferation and migration. Based on the aforementioned data, we hypothesized that miR-134 may be involved in the NCK1-AS1-mediated proliferation and migration of cervical cancer cells. To confirm this hypothesis, HeLa and $\mathrm{SiHa}$ cells were co-transfected with NCK1-AS1 siRNA together with an NC inhibitor or a miR-134 inhibitor. As indicated in Fig. 5A, miR-134 was significantly downregulated in the siNCK1-AS1 + anti-miR-134 group compared with the miR-134 levels in the siNCK1-AS1 + anti-NC group. The CCK-8 and wound healing assays additionally demonstrated that the proliferation and migration of cervical cancer cells were significantly enhanced in the siNCK1-AS1 + anti-miR-134 group compared with the siNCK1-AS1 + anti-NC group (Fig. 5B-E), indicating that the knockdown of miR-134 impaired the inhibitory effects on cervical cancer cell proliferation and migration induced by NCK1-AS1 downregulation. Taken together, these data suggested that miR-134 was involved in the NCK1-AS1-mediated proliferation and migration of cervical cancer cells.

\section{Discussion}

The regulatory mechanism underlying the effect of NCK1-AS1 in cervical cancer progression remains largely unclear. In the present study, it was identified that 

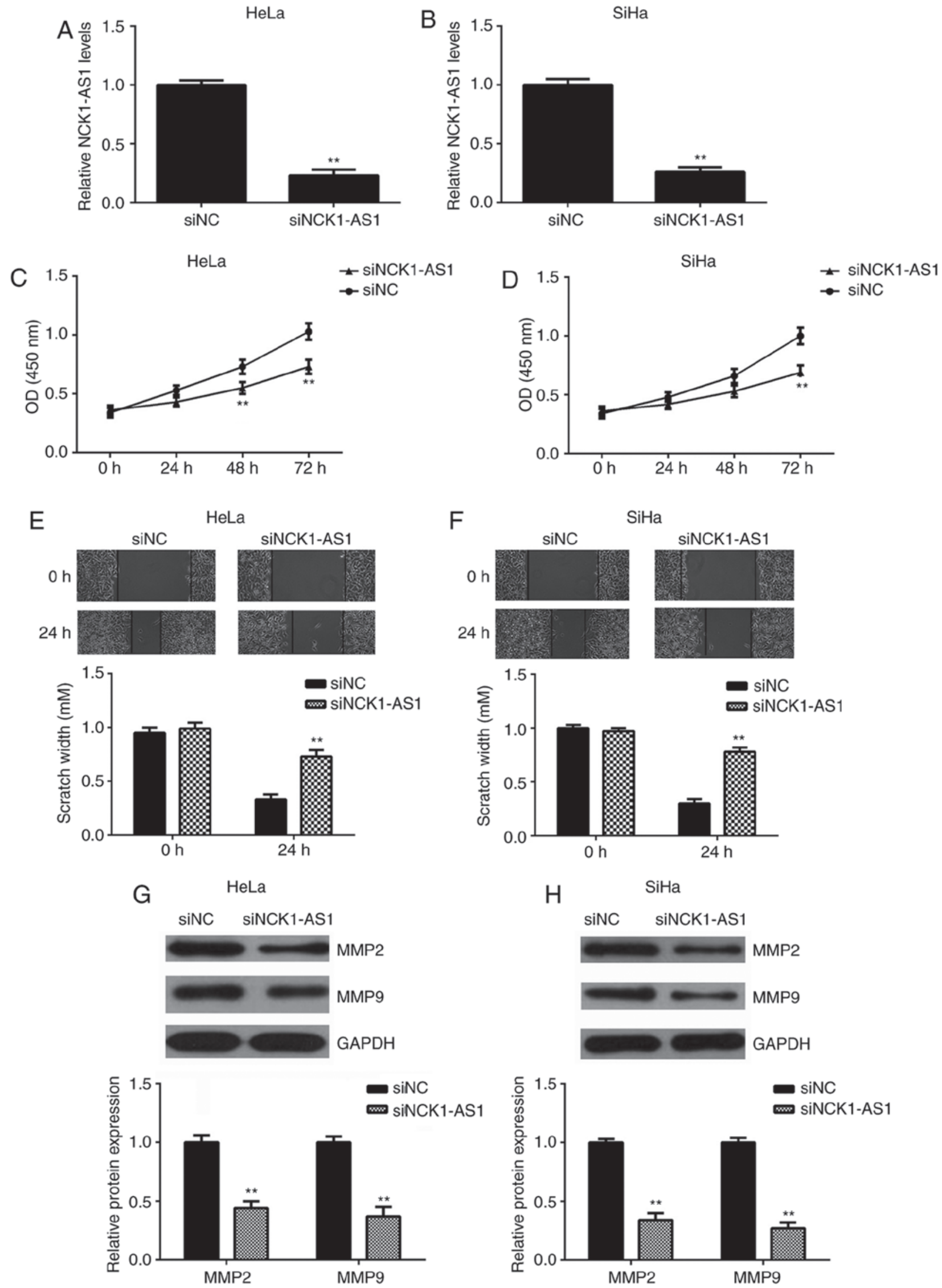

Figure 2. Silencing of NCK1-AS1 inhibits the proliferation and migration of cervical cancer cells. (A and B) HeLa and SiHa cells were transfected with NC siRNA or NCK1-AS1 siRNA, respectively. Following transfection, quantitative polymerase chain reaction was used to examine the expression of NCK1-AS1 in (A) HeLa and (B) SiHa cells. (C and D) A Cell Counting Kit-8 assay was performed to study cell proliferation in (C) HeLa and (D) SiHa cells. (E and F) A wound healing assay was conducted to study cell migration in (E) HeLa and (F) SiHa cells. (G and H) Western blot analysis was conducted to examine the protein expression of MMP-2 and MMP-9. GAPDH was used as an internal reference. ${ }^{* *} \mathrm{P}<0.01$ vs. siNC. NCK1-AS1, NCK1-antisense 1; siRNA, small interfering RNA; NC, negative control; MMP, matrix metalloproteinase; OD optical density. 

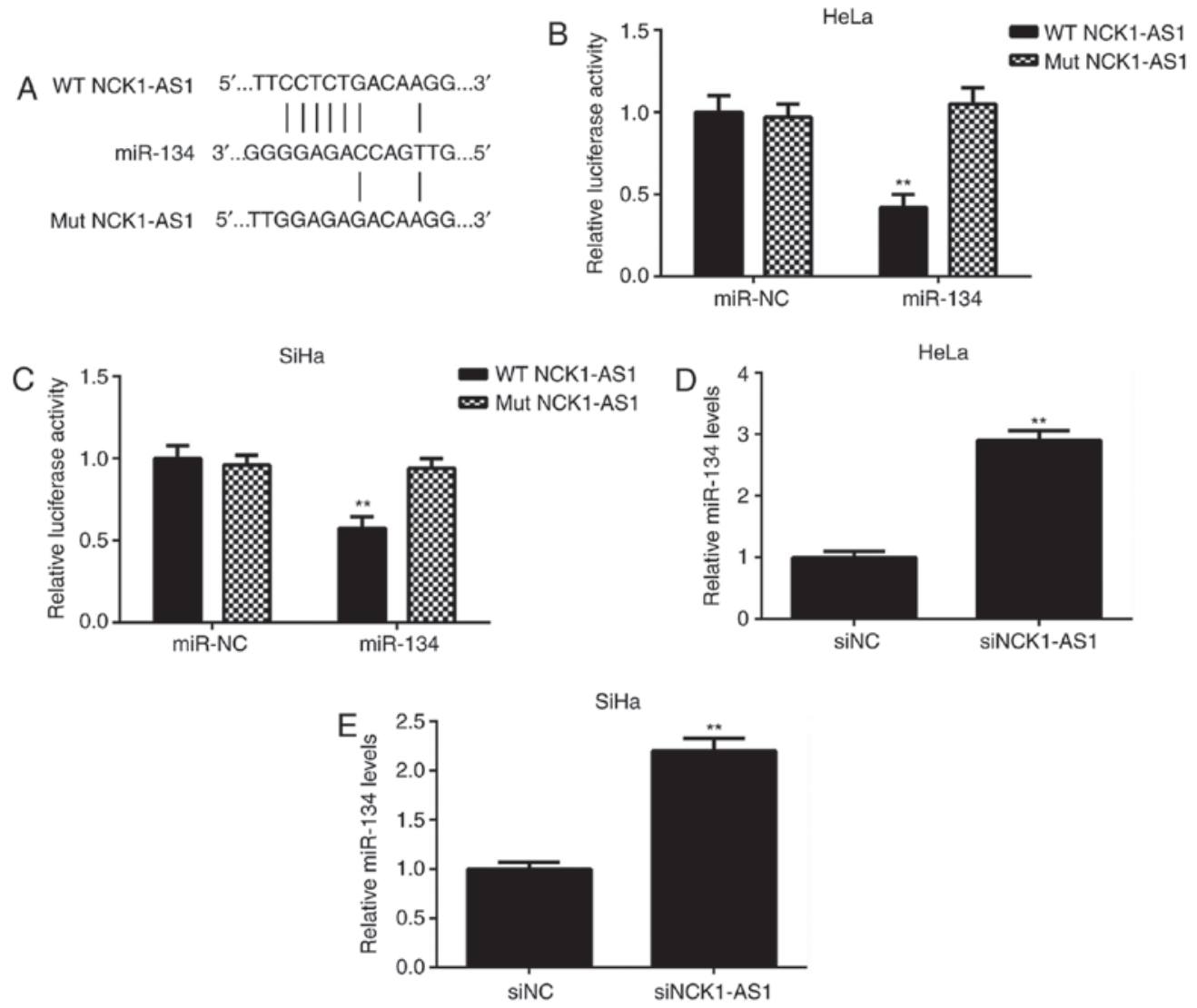

Figure 3. NCK1-AS1 gene contains an miR-134 binding site in cervical cancer cells. (A) The WT and MT miR-134 binding sites in NCK1-AS1. (B and C) The luciferase reporter gene assay indicated that transfection with miR-134 mimic decreased the luciferase activity of the WT NCK1-AS1 reporter plasmid, while it had no effect on the luciferase activity of the MT NCK1-AS1 reporter plasmid in (B) HeLa and (C) SiHa cervical cancer cells. ${ }^{* * *} \mathrm{P}<0.01$ vs. miR-NC. (D and E) Knockdown of NCK1-AS1 significantly increased the expression levels of miR-134 in (D) HeLa and (E) SiHa cervical cancer cells. " $\mathrm{P}<0.01$ vs. siNC. NCK1-AS1, NCK1-antisense 1; miR-134, microRNA-134; WT, wide type; MT, mutant type; NC, negative control; si, small interfering RNA.
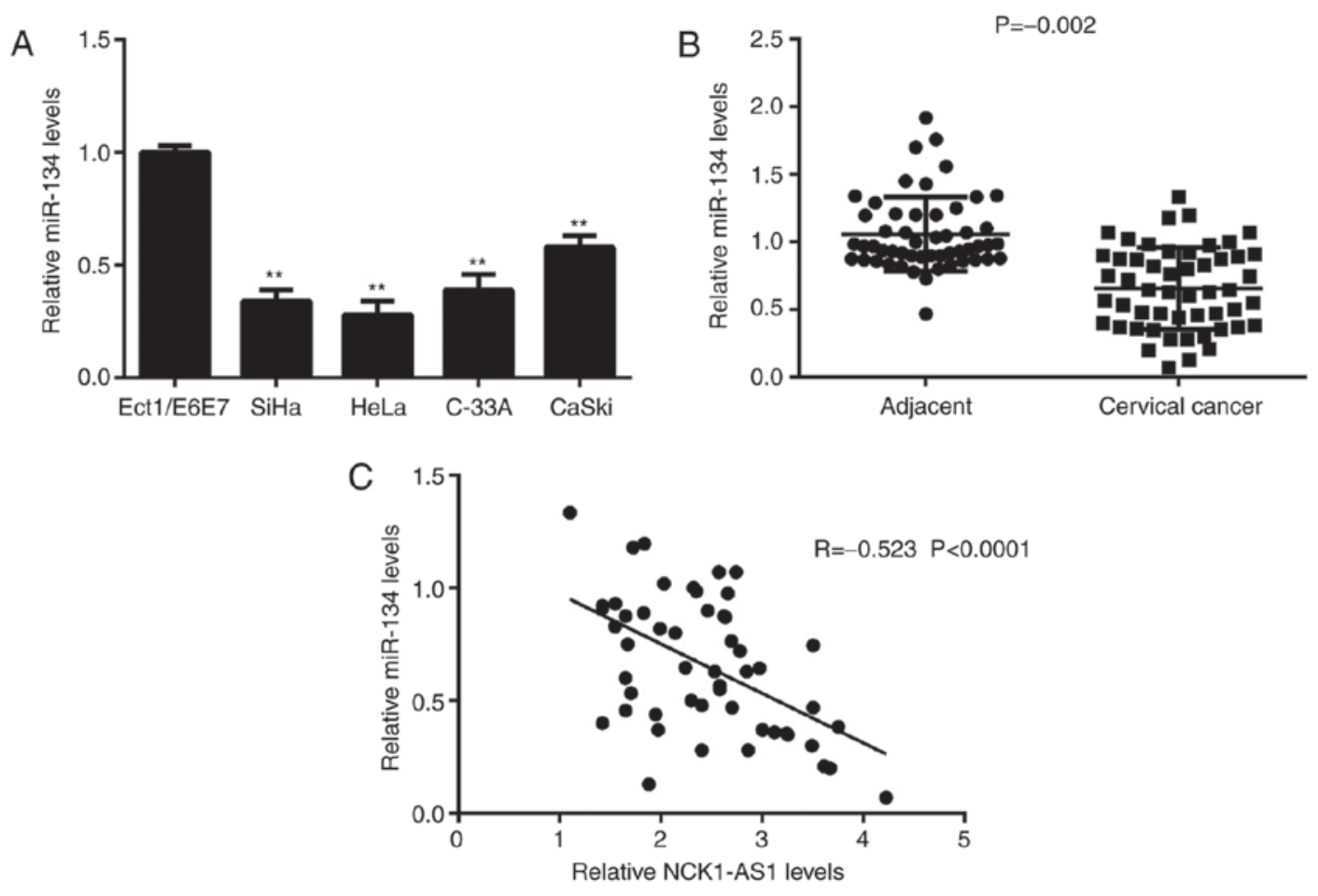

Figure 4. Downregulation of miR-134 is inversely correlated with the upregulation of NCK1-AS1 in cervical cancer. (A) The miR-134 levels were significantly downregulated in cervical cancer cell lines compared with the miR-134 levels in Ect1/E6E7 cells. ${ }^{* *} \mathrm{P}<0.01$ vs. Ect1/E6E7. (B) miR-134 was also significantly upregulated in cervical cancer tissues compared with the miR-134 levels in adjacent normal tissues. (C) The expression levels of miR-134 were inversely correlated with the NCK1-AS1 levels in cervical cancer tissues. miR-134, microRNA-134; NCK1-AS1, NCK1-antisense 1. 

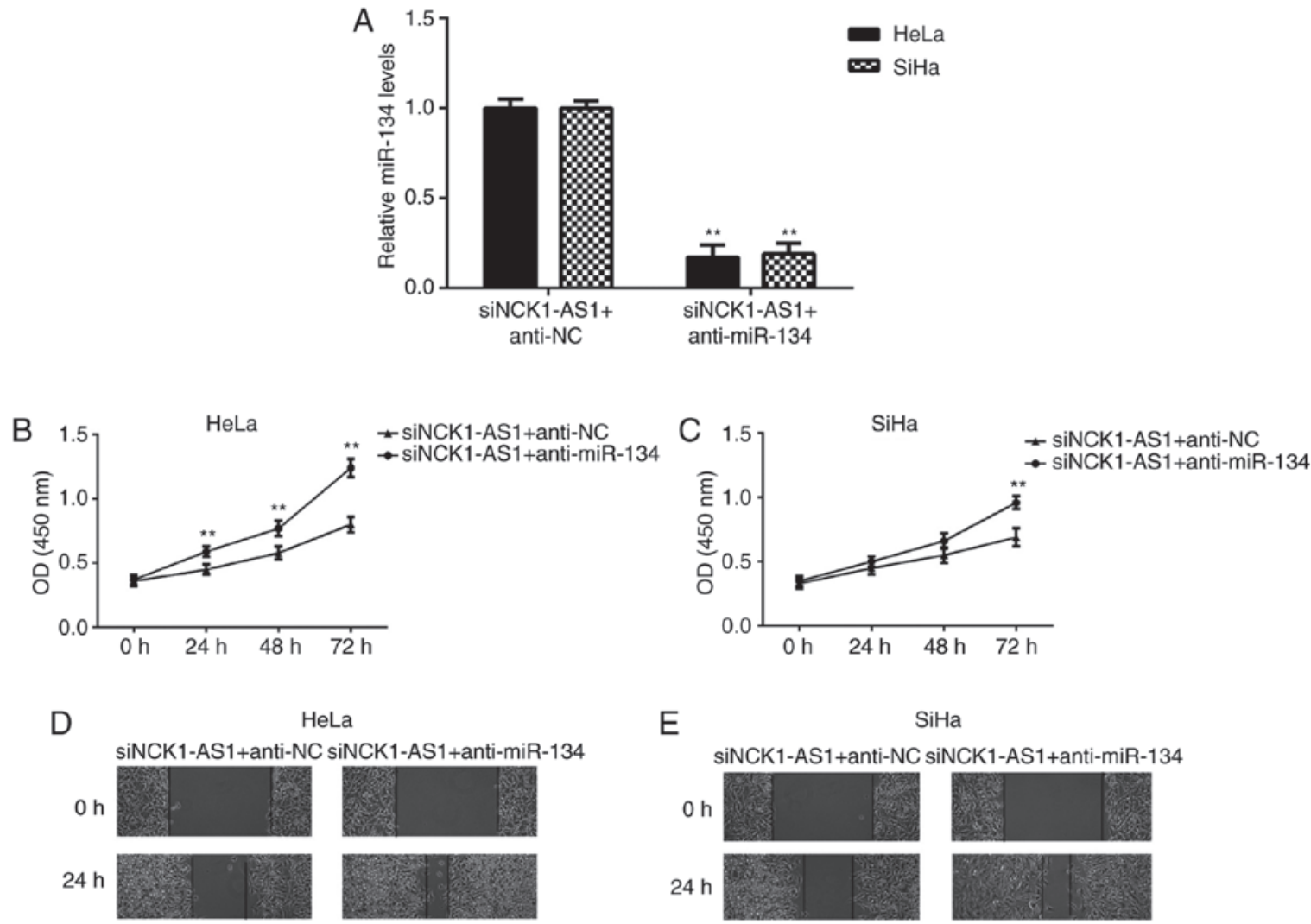

E

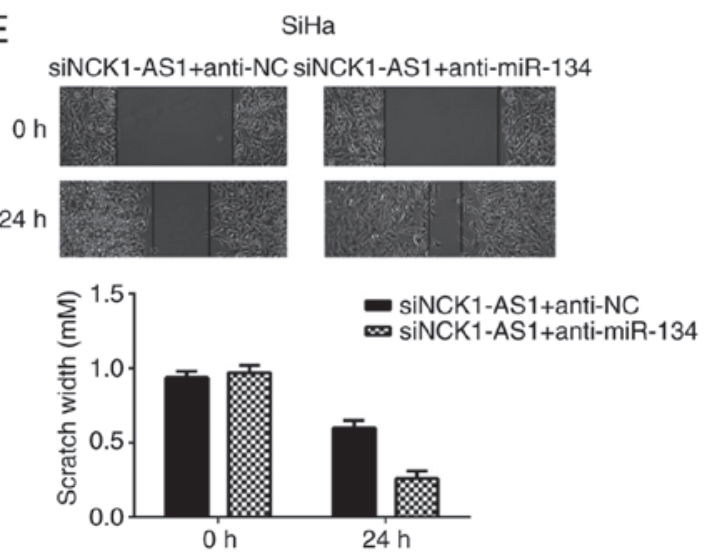

Figure 5. Silencing of miR-134 expression impairs the suppressive effects of NCK1-AS1 inhibition on cervical cancer cell proliferation and migration HeLa and SiHa cells were transfected with NCK1-AS1 siRNA, together with miR-134 inhibitor or NC inhibitor, respectively. (A) Quantitative polymerase chain reaction was used to examine the expression of NCK1-AS1 following transfection. (B and C) A Cell Counting Kit-8 assay was used to study cell proliferation in (B) HeLa and (C) SiHa cells. (D-E) A wound healing assay was conducted to study cell migration in (D) $\mathrm{HeLa}$ and (E) $\mathrm{SiHa}$ cells. ${ }^{* *} \mathrm{P}<0.01 \mathrm{vs}$. siNCK1-AS1+anti-NC. miR-134, microRNA-134; NCK1-AS1, NCK1-antisense 1; siRNA, small interfering RNA; NC, negative control; OD, optical density.

NCK1-AS1 was significantly upregulated in cervical cancer tissues compared with the NCK1-AS1 expression in adjacent non-tumour tissues. High expression levels of NCK1-AS1 were associated with tumour progression and poor prognosis in patients with cervical cancer. Silencing of NCK1-AS1 expression decreased cervical cancer cell proliferation and migration, and decreased the protein expression levels of MMP-2 and MMP-9. The results of the luciferase reporter gene assay indicated that an miR-134 binding site was contained within the NCK1-AS1 gene. miR-134 was significantly downregulated in cervical cancer tissues compared with the miR-134 levels in adjacent non-tumour tissues, and its expression levels were inversely correlated with the expression levels of NCK1-AS1 in cervical cancer tissues. Knockdown of miR-134 attenuated the inhibitory effects on the proliferation and migration of cervical cancer cells induced by NCK1-AS1 downregulation.

Recently, a number of IncRNAs have been suggested to be significantly deregulated in cervical cancer; some of these lncRNAs are associated with tumour progression and poor prognosis $(20,21)$. For example, IncRNA HOX transcription antisense RNA is significantly upregulated in cervical cancer and promotes cancer progression by increasing the expression of Bcl-2 via the inhibition of miR-143-3p (22). The lncRNA Pvt-1 oncogene facilitates the proliferation, migration and invasion of cervical cancer cells by negatively regulating miR-424 (21). In the present study, an upregulation of NCK1-AS1 was observed in cervical cancer, and high expression levels of NCK1-AS1 were associated with lymph node metastasis, advanced clinical stage and poor prognosis of patients. These data suggested that NCK1-AS1 upregulation may contribute to cervical cancer progression. The role of NCK1-AS1 in cervical cancer growth and metastasis in vitro was also studied. To knock down the expression of NCK1-AS1, cervical cancer cells were transfected with NCK1-AS1-specific siRNA. The results demonstrated that the silencing of NCK1-AS1 significantly inhibited the proliferation and migration of cervical cancer cells, suggesting that NCK1-AS1 may serve a promotive role in cervical cancer growth and metastasis. 
The downstream miRNAs of NCK1-AS1 was then studied in cervical cancer cells, and bioinformatics analysis predicted that miR-134 and NCK1-AS1 had binding sites in cervical cancer cells. Previous studies have demonstrated that miR-134 serves an oncogenic or a tumour suppressive role in different types of human cancer: For example, Peng et al (7) revealed that miR-134 targeted PDCD7 to decrease epithelial cadherin expression and enhance oral cancer progression. By contrast, However, to the best of our knowledge, the expression pattern and the detailed role of miR-134 in cervical cancer has not been studied. In the present study, it was identified that the expression of miR-134 was significantly decreased in cervical cancer tissues and cell lines compared with that in adjacent tissues and normal cells. In addition, it was demonstrated that the expression of miR-134 was inversely correlated with NCK1-AS1 expression in cervical cancer tissues, and that silencing of NCK1-AS1 expression increased miR-134 expression in cervical cancer cells. These data suggested that the downregulation of miR-134 may be due to the upregulation of NCK1-AS1 in cervical cancer. It was then revealed that the inhibition of miR-134 impaired the suppressive effects on the proliferation and migration of cervical cancer cells induced by NCK1-AS1 knockdown, which suggested that miR-134 may serve as a downstream effecter of NCK1-AS1 in cervical cancer cells.

In addition to NCK1-AS1, several other lncRNAs have been identified to directly target miR-134 in other cancer types. For example, through the inhibition of miR-134-5p, the IncRNA prostate cancer associated transcript 7 regulated E74 like ETS transcription factor 2 signalling in nasopharyngeal carcinoma (23), and induced poor prognosis and promoted tumourigenesis in non-small-cell lung cancer (24). Therefore, the results of the present study improve the understanding of the LncRNA/miRNA axis in human cancer.

To the best of our knowledge, this is the first study to demonstrate that silencing of NCK1-AS1 expression repressed the proliferation and migration of cervical cancer cells via miR-134, suggesting that NCK1-AS1 may be used as a potential therapeutic target for cervical cancer.

\section{Acknowledgements}

Not applicable.

\section{Funding}

No funding was received.

\section{Availability of data and materials}

All data generated or analysed during this study are included in this published article.

\section{Authors' contributions}

JY and LHu designed the study and wrote the manuscript. XG collected clinical tissues and performed statistical analysis. $\mathrm{LHu}$, LHe and LW performed all experiments. All authors read and approved the final manuscript.

\section{Ethics approval and consent to participate}

This study was approved by the Ethics Committee of Chengdu Women and Children's Central Hospital. Written informed consent was obtained from all patients involved.

\section{Patient consent for publication}

All patients provided written informed consent.

\section{Competing interests}

The authors declare that they have no competing interests.

\section{References}

1. Jemal A, Siegel R, Ward E, Murray T, Xu J, Smigal C and Thun MJ: Cancer statistics, 2006. CA Cancer J Clin 56: 106-130, 2006.

2. Torre LA, Bray F, Siegel RL, Ferlay J, Lortet-Tieulent J and Jemal A: Global cancer statistics, 2012. CA Cancer J Clin 65: 87-108, 2015.

3. King T: HPV, cervical cancer, and you. J Midwifery Womens Health 53: 263-264, 2008

4. Yang WT and Zheng PS: Kruppel-like factor 4 functions as a tumor suppressor in cervical carcinoma. Cancer 118: 3691-3702, 2012.

5. Shen Y, Zhou J, Li Y, Ye F, Wan X, Lu W, Xie X and Cheng X: miR-375 mediated acquired chemo-resistance in cervical cancer by facilitating EMT. PLoS One 9: e109299, 2014.

6. Zhou S, Yu L, Xiong M and Dai G: LncRNA SNHG12 promotes tumorigenesis and metastasis in osteosarcoma by upregulating Notch2 by sponging miR-195-5p. Biochem Biophys Res Commun 495: 1822-1832, 2018.

7. Peng Z, Liu C and Wu M: New insights into long noncoding RNAs and their roles in glioma. Mol Cancer 17: 61, 2018.

8. Xu R, Zhu X, Chen F, Huang C, Ai K, Wu H, Zhang L and Zhao X: LncRNA XIST/miR-200c regulates the stemness properties and tumourigenicity of human bladder cancer stem cell-like cells. Cancer Cell Int 18: 41, 2018.

9. Xiong W, Huang C, Deng H, Jian C, Zen C, Ye K, Zhong Z, Zhao X and Zhu L: Oncogenic non-coding RNA NEAT1 promotes the prostate cancer cell growth through the SRC3/IGF1R/AKT pathway. Int J Biochem Cell Biol 94: 125-132, 2018.

10. Xiao Z, Qu Z, Chen Z, Fang Z, Zhou K, Huang Z, Guo X and Zhang Y: LncRNA HOTAIR is a prognostic biomarker for the proliferation and chemoresistance of colorectal cancer via miR-203a-3p-mediated Wnt/ $\beta$-catenin signaling pathway. Cell Physiol Biochem 46: 1275-1285, 2018.

11. Sun Y, Jin JG, Mi WY, Zhang SR, Meng Q and Zhang ST: Long noncoding RNA UCA1 targets miR-122 to promote proliferation, migration, and invasion of glioma cells. Oncol Res 26: 103-110, 2018.

12. Lv L, Jia JQ and Chen J: The lncRNA CCAT1 upregulates proliferation and invasion in melanoma cells via suppressing miR-33a. Oncol Res 26: 201-208, 2018.

13. Zhu H, Zheng T, Yu J, Zhou L and Wang L: LncRNA XIST accelerates cervical cancer progression via upregulating Fus through competitively binding with miR-200a. Biomed Pharmacother 105: 789-797, 2018.

14. Zhu J, Shi H, Liu H, Wang X and Li F: Long non-coding RNA TUG1 promotes cervical cancer progression by regulating the miR-138-5p-SIRT1 axis. Oncotarget 8: 65253-65264, 2017.

15. Li H, Jia Y, Cheng J, Liu G and Song F: LncRNA NCK1-AS1 promotes proliferation and induces cell cycle progression by crosstalk NCK1-AS1/miR-6857/CDK1 pathway. Cell Death Dis 9: 198, 2018.

16. Bartel DP: MicroRNAs: Genomics, biogenesis, mechanism, and function. Cell 116: 281-297, 2004.

17. Pan JY, Zhang F, Sun CC, Li SJ, Li G, Gong FY, Bo T, He J, Hua RX, Hu WD, et al: miR-134: A human cancer suppressor? Mol Ther Nucleic Acids 6: 140-149, 2017.

18. Liu Y, Sun Y and Zhao A: MicroRNA-134 suppresses cell proliferation in gastric cancer cells via targeting of GOLPH3. Oncol Rep 37: 2441-2448, 2017. 
19. Livak KJ and Schmittgen TD: Analysis of relative gene expression data using real-time quantitative PCR and the 2(-Delta Delta C(T)) method. Methods 25: 402-408, 2001.

20. Zhang JJ, Wang DD, Du CX and Wang Y: Long noncoding RNA ANRIL promotes cervical cancer development by acting as a sponge of miR-186. Oncol Res 26: 345-352, 2018.

21. Gao YL, Zhao ZS, Zhang MY, Han LJ, Dong YJ and Xu B: Long Non-coding RNA PVT1 facilitates cervical cancer progression via negative regulating of miR-424. Oncol Res 25: 1391-1398, 2017.

22. Liu M, Jia J, Wang X, Liu Y, Wang C and Fan R: Long non-coding RNA HOTAIR promotes cervical cancer progression through regulating BCL2 via targeting miR-143-3p. Cancer Biol Ther 19: 391-399, 2018.
23. Liu Y, Tao Z, Qu J, Zhou X and Zhang C: Long non-coding RNA PCAT7 regulates ELF2 signaling through inhibition of miR-134-5p in nasopharyngeal carcinoma. Biochem Biophys Res Commun 491: 374-381, 2017.

24. Liu Q, Wu Y, Xiao J and Zou J: Long Non-coding RNA prostate cancer-associated transcript 7 (PCAT7) induces poor prognosis and promotes tumorigenesis by inhibiting mir-134-5p in non-small-cell lung (NSCLC). Med Sci Monit 23: 6089-6098, 2017. 